\title{
Anatomically Constrained Surface Parameterization for Cortical Localization
}

\author{
C. Clouchoux ${ }^{1}$, O. Coulon ${ }^{1}$, D. Rivière ${ }^{2}$, A. Cachia $^{2}$, \\ J.-F. Mangin ${ }^{2}$, and J. Régis ${ }^{3}$ \\ 1 Laboratoire LSIS, UMR 6168, CNRS, Marseille, France \\ ${ }^{2}$ Equipe UNAF, SHFJ, CEA/DSV, Orsay, France \\ 3 Service de Neurochirurgie Fonctionnelle et Stéréotaxique, \\ Marseille, France
}

\begin{abstract}
We present here a method that aims at defining a surfacebased coordinate system on the cortical surface. Such a system is needed for both cortical localization and intersubject matching in the framework of neuroimaging. We propose an automatic parameterization based on the spherical topology of the grey/white matter interface of each hemisphere and on the use of naturally organized and reproducible anatomical features. From those markers used as initial constraints, the coordinate system is propagated via a PDE solved on the cortical surface.
\end{abstract}

\section{Introduction}

In the context of inter-subject brain data matching and localization, most methods deal with 3-dimensional images and consider the problem as a registration one, known as spatial normalization (e.g. 7]). Nevertheless there is great interest in analyzing data projected on the cortical surface [6]. The three-dimensional space does not provide any information about cortical organization. In this context, matching cortical surfaces implies facing several problems, the main one being the lack of an implicit coordinate system, such as the voxel grid in 3 dimensions. Instead of defining spatial normalization as a registration process, we can approach it in terms of localization. Few methods aim at building a surface referential by parameterizing the cortical surface in a reproducible way [6 16 5]. The method presented in [6] relies on the mapping of the cortical surface to a sphere. Resulting surfaces are aligned using a convexity measure, without any information on the cortical organization. In [16], the method presented allows a parameterization of the cortical surface from a geometric atlas, using a few anatomical constraints, but implying a deformation of the cortex, and a manual registration of the markers. Both methods require a warping of the surface to a sphere, which implies distortion on the shape and the distances on the surface.

In this framework, we propose here a method to automatically provide an anatomically meaningful parameterization, based on the definition of invariant and organized anatomical features, and which does not require any warping of the surface 44. The paper is organized as follow : in section 2 we present the cortical anatomy theories we based our method on. Section 3 presents the method itself. Result and discussion are then detailed in section 4 . 


\section{Anatomical Background}

The major problem in studying human brains is the great inter-subject variability. The pattern of a sulcus, even a major one, can have a different shape or different topology from one brain to another. One of the reasons of this variability is thought to be the result of the cortex folding process [112 16 14. To provide invariant features, we have to understand the sulci organization over the cortical surface 81614. In [16, a geometric model of the cortical surface is proposed, which suggests an orthogonal organization scheme of main sulci. The idea of defining a geometric model of the cortical surface was introduced by [15]. In 13, a new approach is proposed to explain the sulci organization: the sulcal roots model. A key idea of this model is that the spatial stability of the deep sulcal cortex is greater than that of a superficial image of the sulci. This point led us to consider not only sulci, but deeply buried subparts of sulci as well, in order to generate the most generic set of anatomical features possible. The previous model provides this information: indivisible units, the so-called sulcal roots, corresponding to the first folding locations during antenatal life. This model raised the hypothesis of the presence of two major orthogonal directions of anatomical organization in the cerebral cortex. Figure 1 shows the some folding process results. Considering that and the spherical topology of each hemisphere led us to define a longitude/latitude coordinate system. Each sulcal root is surrounded by two meridians and two parallels of this gyral organization.

Although each sulcal root defines a cross point between longitude and latitude coordinates, several sulcal roots may be aligned on one sulcus, as shown in figure 1. The resulting fold defines then one coordinate, either meridian or parallel. Cortical folds corresponding to this criteria are thus considered as stable and reproducible markers. A consequence is that from the sulci point of view, sulcal roots are "specialized" in one coordinate. Hence, if a fold is considered to be a longitude constraint, it cannot and will not be used as a latitude constraint.
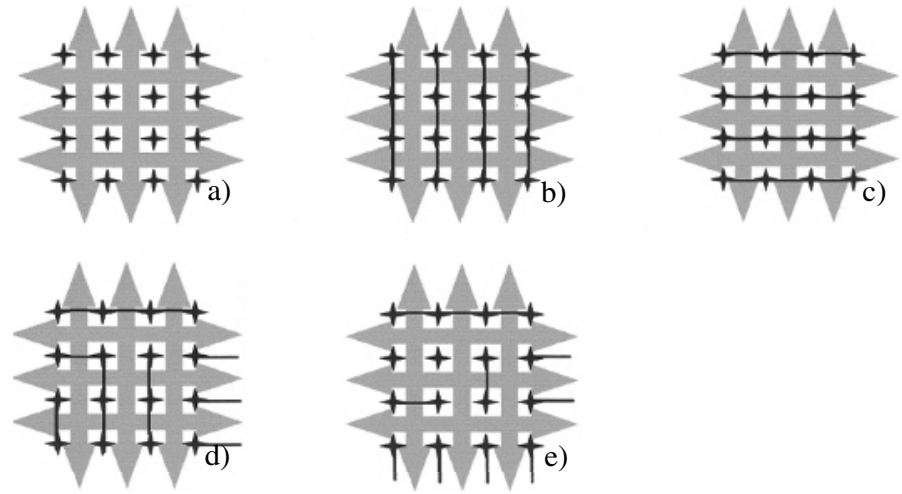

Fig. 1. Meridian/parallel organization around the sulcal roots. a) fœtal stage, b-e) different results of the folding process [13. 
The correlation between sulcal-based representation and gyral-based representation has been established by 2]. In this context, sulci are considered as indicators of the meeting lines buried in the fold's depth between the two neighboring gyri. To define each gyrus borders, a set of sulci has been chosen, thought to be reliable and reproducible through individuals. In our work, a comparable process has been done. To obtain a reliable coordinate system over the cortical surface, we need to define some anatomical markers, present in every brain. The goal is to provide a generic set of those markers, which can be used as landmarks on every nonpathological mature brain. The next section details the choice of such constraints, and the algorithm used to obtain a parameterization of the whole cortical surface.

\section{Method}

The outline of our method is to automatically build a complete parameterization, in a longitude/latitude manner, starting from a few anatomical markers and propagating a coordinate system from those original constraints over the whole cortical surface of each hemisphere. All processings presented below are performed on the original cortical surface. Brains shown in figures are inflated for visualization purpose only.

\subsection{Anatomical Markers}

The first step of our method is the selection of anatomical markers. As explained above, a set of sulci, considered to be reproducible, can be defined. The set we built up is approximatively the same than the one used for the gyral-based representation in [2]. From MR anatomical images, a preprocessing stage provides a triangulation of the cortex hemisphere endowed with a spherical topology [910], corresponding to the interface between the gray and the white matter. From those images, an automatic recognition process provides the identification of the main sulci [14] (see figure 2).

At this stage of the process, the set of markers is chosen. To have a complete longitude/latitude coordinate system, two poles and an origin meridian are required. Latitude will be propagated from one pole to another, and longitude from one side of the origin meridian to another [1. The poles are chosen as defined in [12. One is the Insula. This area is the first folding zone appearing during the human brain growth. The other is an extension of the projection of the Corpus Callosum. The origin meridian is the one corresponding to the Central Sulcus. Indeed, this fold is one of the most stable sulci across individuals, and almost links the two poles together.

The sulci $3 \mathrm{D}$ representation is then used to get the projection of the bottom sulcal line on the cortical surface [2] of the set of sulci selected before, as shown in figure 2 .

The diffusion process needs a complete origin meridian, i.e. linking one pole to another. The Central Sulcus projection is extended, using the shortest geodesic distance between the poles and the extremities of the sulcus projection. 

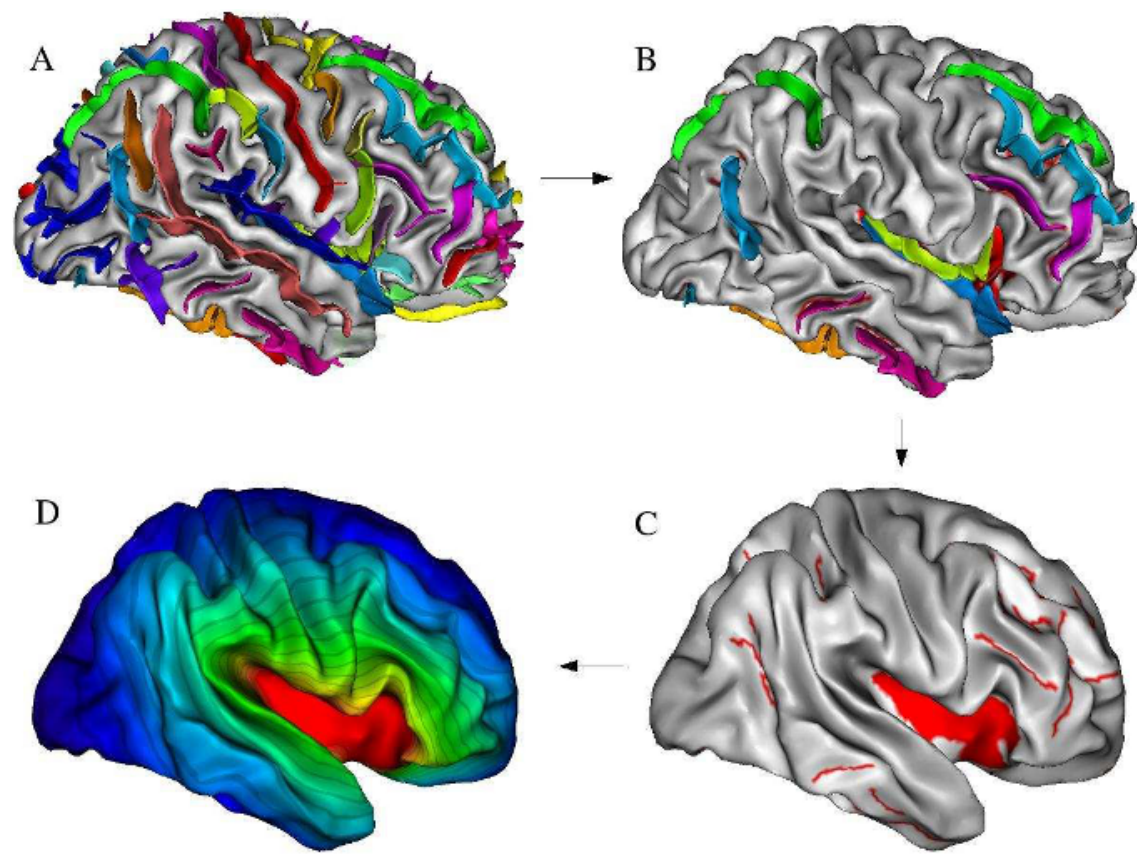

Fig. 2. Illustration of the main steps of the coordinates propagation: (A) Right hemisphere with all its sulci (each color represents a label). (B) Set of chosen constraints for latitude. (C) Constraints projection on smoothly inflated cortical mesh. (D) Resulting latitude coordinates (All visualizations made with free package Brainvisa/Anatomist - http://brainvisa.info).

Once we have defined the whole constraints set, including poles and origin meridian, each projection is attributed a constant longitude (meridians) or latitude (parallels). As we want an homogeneous spreading of the coordinates over the cortical surface, attention must be given to the coordinates values attributed to the constraints. A last step consists in removing some small branches of the projected sulci, in order to guaranty that each connected component is a simply connected object for the mesh topology.

\subsection{Coordinates Propagation}

The aim of the parameterization is to obtain a global coordinate system that complies with the initial anatomical constraints. To get such a result, we propagate the coordinates from the constraints. Markers are used as sources of a surfacic heat-equation diffusion process [1] that drives the propagation of both longitude and latitude over the whole hemisphere surface. Equation 1 shows a continuous heat diffusion process, where $\frac{\partial I(\vec{r}, t)}{\partial t}$ represents the heat diffusion on the surface, $t$ the time, $\nabla^{2}$ the laplacian operator and $K$ a constant conduction parameter. 


$$
\frac{\partial I(\boldsymbol{r}, t)}{\partial t}=K \nabla^{2} I(\boldsymbol{r}, t)
$$

As we are on a discrete domain, we use a finite element method to estimate $\nabla^{2} I[3]$ and get the iterative numerical scheme shown in equation 2]:

$$
I(\boldsymbol{r}, t+\Delta t)=I(\boldsymbol{r}, t)+\Delta t K \hat{\nabla}^{2} I(\boldsymbol{r}, t),
$$

where $\hat{\nabla}^{2} I(\boldsymbol{r}, t)$ is a local estimate of the laplacian at time $t$. Stability is reached when $\hat{\nabla}^{2} I(\boldsymbol{r}, t) \rightarrow 0$. As presented in [3], $\hat{\nabla}^{2} I$ is defined on each node of the surface mesh as a weighted sum of the nodes neighbours, taking into account the local geometry of the surface. Longitude and latitude are propagated separately, in an iterative process. First, the surface is initialized with the contraints and their mean value on the rest of the surface. The process then starts, iteratively updating the value at every node of the surface, except on the constraints, defined
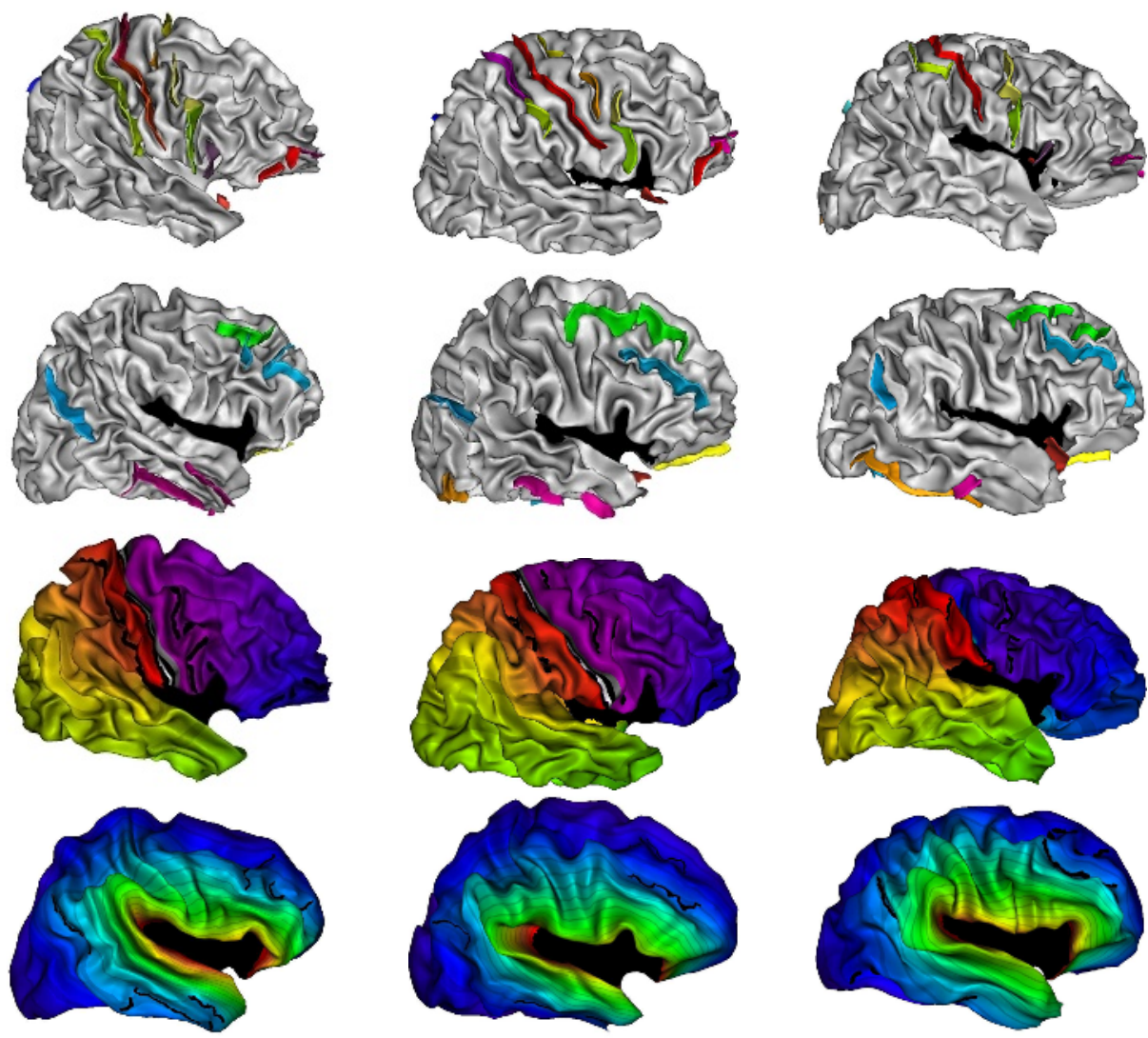

Fig. 3. Results of the coordinates propagation on six different brains. (Top row) Constraints for longitude and (second row) latitude. Resulting diffusion (constraints and isoparameter lines) for longitude (third row) and latitude (bottom row). 
as constant heat sources. The result is obtained once the stability is reached, i.e. when $\hat{\nabla}^{2} I \rightarrow 0$, leading to an homogeneous distribution of the coordinates values between the constraints.

\section{Results and Discussion}

We tested our algorithm on six nonpathological mature brains, taken from the ICBM database. Figure 3 shows the initial constraints we used and the resulting coordinate system, on the six brains. An exemple of detailed result is shown on figure 4

The experiment led on 6 different brains shows a good behavior of the coordinate propagation. As we see on figure 4, iso-density lines of the coordinates system follows the global geometry of the surface, and locally complies with the anatomical constraints, included as axes over the surface. Coordinates are homogeneously spred all over the surface, between the constraints. Figure 5 shows in detail how the parameterization complies with the markers.
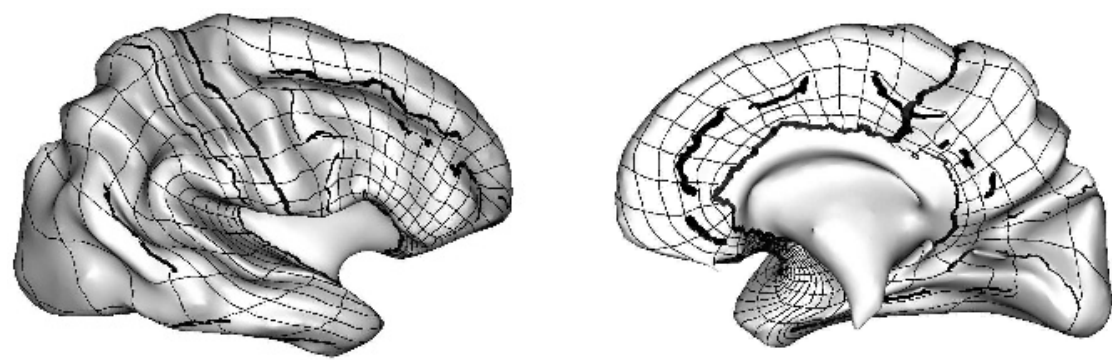

Fig. 4. Global obtained coordinate system on a right hemisphere, with resulting isodensity lines and latitude and longitude constraints
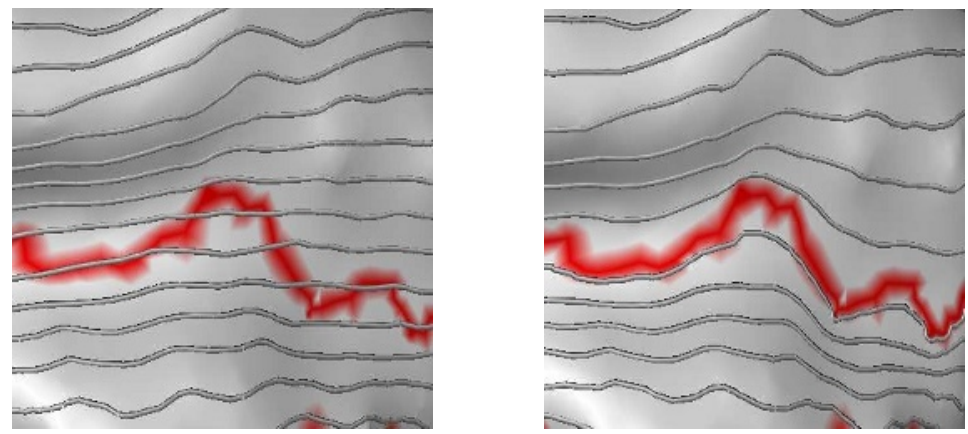

Fig. 5. Iso-density line complying with a sulcus projection. (left) Zoom on propagation result done without any constraint. (right) Propagation done with constraint, complying with the constraint (superimposed in red in both cases). 
One of the problems we encounter is that parallels and meridians are propagated separately from each other. Because of this, we cannot guarantee theoretically the unicity of coordinate couples, although this problem can only be local.

We also must be aware of the influence of the inacurracy of some steps of our process, e.g. the automatic sulci recognition.

Currently, anatomical markers are subparts of reproducible sulci. A great improvement of our method would be the refinement of the definition of the markers, to match the sulcal roots model the best we could. That means we must be able to have a projection of sulcal roots. Such a process needs an important work on cortical anatomy, and is being studied at the moment.

\section{Conclusion and Further Work}

In this paper, we defined a process that aims at implementing an orthogonal coordinate system on every cortical surface. This parameterization relies on the theoretical orthogonal organization of the sulci. The algorithm used gives a global parameterization of the surface, that complies with anatomical markers.

From this work, several lines of research arise: refinement of the anatomical features used, the guarantee of the unicity of the parameterization which we are currently trying to solve by coupling the diffusion of longitude and latitude, and validation in a neuroimaging experimental context.

Our work could be also extended to a functional description of the surface, based on cortical folds. Other future applications are surface morphometry, or localization for the integration of modalities such as EEG and MEG.

\section{References}

1. Brechbuhler, C., Gerig, G., Kobler, O.: Parameterization of closed surfaces for 3d shape description. Computer Vision and Image Understanding 61 (1995) 154-170

2. Cachia, A., Mangin, J.F., Rivière, D., Papadopoulos-Orfanos, D., Kherif, F., Bloch, I., Régis, J.: A generic framework for parcellation of the cortical surface into gyri using geodesic voronoi diagrams. Medical Image Analysis 7 (2003) 403-416

3. Chung, M., Taylor, J.: Diffusion smoothing on brain surface via finite element method. In: IEEE International Symposium on Biomedical Imaging (ISBI). (2004)

4. Clouchoux, C., Coulon, O., Cachia, A., Rivière, D., Mangin, J.F., Régis, J.: Towards an anatomically meaningful parameterization of the cortical surface. In Barillot, C., Haynor, D., Hellier, P., eds.: Proc. of MICCAI'04. LNCS, Saint-Malo, France, Springer Verlag (2004)

5. Essen, D.V., Drury, H.: Structural and functional analyses of human cerebral cortex using a surface-based atlas. The Journal of Neuroscience 17 (1997) 7079-7102

6. Fischl, B., Sereno, M., Tootell, R., Dale, A.: Cortical surface-based analysis, ii: Inflation, flattening, and a surface-based coordinate system. NeuroImage $\mathbf{9}$ (1999) 195-207

7. Friston, K., Ashburner, J., Poline, J., Frith, C., Heather, J., Frackowiak, R.: Spatial registration and normalisation of images. In: Human Brain Mapping. Volume 2. (1995) 165-189 
8. Lohmann, G., von Cramon, Y.: Automatic labeling of the human cortical surface using sulcal basins. Medical Image Analysis 4 (1999) 179-188

9. Mangin, J.F., Frouin, V., Bloch, I., Régis, J., López-Krahe, J.: From 3D magnetic resonance images to structural representations of the cortex topography using topology preserving deformations. Journal of Mathematical Imaging and Vision $\mathbf{5}$ (1995) 297-318

10. Mangin, J.F., Régis, J., Frouin, V.: Shape bottlenecks and conservative flow systems. In Press, I.C.S., ed.: IEEE Work. MMBIA, San Francisco, CA (1996) 319-328

11. Ono, M., Kubik, S., Abernathey, C.: Atlas of the cerebral sulci. Thieme Medical Publishers, Inc., New York (1990)

12. Régis, J., Mangin, J.F., Frouin, V., Sastre, F., Peragut, J.C., Samson, Y.: Generic model for the localization of the cerebral cortex and preoperative multimodal integration in epilepsy surgery. Stereotactic and Functional Neurosurgery 65 (1995) $72-80$

13. Régis, J., Mangin, J., Ochiai, T., Frouin, V., Rivière, D., Cachia, A., Tamura, M., Samson, Y.: Sulcal roots generic model: a hypothesis to overcome the variability of the human cortex folding patterns. Neurol Med Chir 45 (2005) 1-17

14. Rivière, D., Mangin, J., Papadopoulos-Orfanos, D., Martinez, J., Frouin, V., Régis, J.: Automatic recognition of cortical sulci of the human brain using a congregation of neural networks. Medical Image Analysis 6 (2002) 77-92

15. Todd, P.: A geometric model for the cortical folding pattern of simple folded brains. J Theor Biol 97 (1982) 529538

16. Toro, R., Burnod, Y.: Geometric atlas: modeling the cortex as an organized surface. NeuroImage 20 (2003) 1468-1484 